\title{
TARMED - Änderungen per 1. Oktober 2014
}

Ernst Gählera,

Roger Scherrer ${ }^{b}$

a Dr. med., Vizepräsident der FMH, Verantwortlicher Departement Ambulante Tarife und Verträge Schweiz

b FMH, Leiter Abteilung Ambulante Tarife und Verträge Schweiz
FMH / Abteilung Ambulante Tarife und Verträge Schweiz Frohburgstrasse 15

CH-4600 Olten

Tel. 0313591230

Fax 0313591238

tarife.ambulant[at]fmh.ch
Der Bundesrat hat am 20. Juni 2014 die Verordnung über die Anpassung von Tarifstrukturen in der Krankenversicherung erlassen, die gemäss aktuellem Verordnungstext am 1. Oktober 2014 in Kraft treten wird. Die folgenden Ausführungen informieren Sie über die damit verbundenen Änderungen in der Tarifstruktur TARMED und die wichtigsten Fakten. Betreffend kurzfristige Entwicklungen informieren wir Sie auf unserer Webseite sowie per E-Mail via Ihre Tarifdelegierten und Präsidenten der medizinischen Fachgesellschaften bzw. der kantonalen Ärztegesellschaften.

\section{Inhalt der Verordnung}

Die Verordnung macht folgende Vorgaben:

\section{Neue Tarifposition $00.0015+$ Zuschlag für hausärztliche Leistungen in der Arztpraxis}

- Abrechenbar durch Ärzte mit Weiterbildungstiteln Allgemeine Innere Medizin, Kinder- und Jugendmedizin sowie Praktischer Arzt. Auch abrechenbar durch Doppeltitelträger.

- Zuschlagsleistung zur Tarifposition 00.0010

- Bewertung: 10 Taxpunkte ärztliche Leistung (abrechenbar $1 \times$ pro Tag und Patient)

- Darf nur im Zusammenhang mit der Erbringung hausärztlicher Leistungen abgerechnet werden.

- Darf nicht von ambulanten Diensten von Spitälern abgerechnet werden.
Die Unfallversicherer, die Invalidenversicherung und die Militärversicherung haben sich jedoch dieser Lösung angeschlossen.

\section{Technische Umsetzung mit den Tarifpartnern}

Aufgrund der teilweise recht vagen Formulierungen in der Verordnung hat sich die technische Umsetzung der Verordnung als schwierig erwiesen. Die Tarifpartner FMH, H+, MTK, curafutura und santésuisse hatten sich betreffend die tariftechnische Umsetzung in den meisten Punkten geeinigt. In Absprache mit allen Tarifpartnern wurde die Interpretation der neuen Tarifposition 00.0015 durch die Tarifpartner folgendermassen präzisiert:

Darf nur im Zusammenhang mit der Erbringung von hausärztlichen Leistungen abgerechnet werden, und wenn dem Patienten am selben Tag keine spezialärztlichen Leistungen durch den gleichen Leistungserbringer verrechnet werden.

Strittig war bei der Umsetzung die Frage, ob und wie hausärztliche Leistungen von den übrigen TARMED-Leistungen abgegrenzt werden können.

Aus Sicht der FMH und der Hausärzte Schweiz gibt das heute gültige Dignitätskonzept grundsätzlich in genügendem Masse vor, welche Ärztegruppen die neue Leistung 00.0015 abrechnen dürfen. Ausserdem spielt der Kontext der Leistungserbringung eine wichtige Rolle. Die Kostenträgerverbände MTK, santésuisse und curafutura haben beschlossen, ihre Rechnungsprüfung basierend auf einer Gruppe

\section{«Aufgrund der teilweise recht vagen Formulierungen in der Verordnung hat sich die technische Umsetzung der Verordnung als schwierig erwiesen.»}

\section{Kürzung der Taxpunkte der technischen} Leistung in den Kapiteln 04, 05, 08, 15, 17, 19, 21, 24, 31, 32, 35, 37, 39 um 8,5 Prozent

Das TARMED-Kapitel 29 «Schmerztherapie» ist - im Gegensatz zum Verordnungsentwurf - in der definitiven Version der Verordnung nicht mehr von den Kürzungen betroffen.

\section{Geltungsbereich}

Es handelt sich um eine Verordnung zum Krankenversicherungsgesetz. von Tarifpositionen vorzunehmen, welche mit der neuen Position 00.0015 abgerechnet werden dürfen. Wir möchten an dieser Stelle darauf hinweisen, dass diese Gruppe einseitig von den Kostenträgern gebildet und nicht von der FMH und «Hausärzte Schweiz» verabschiedet wurde.

Ein Tarifbrowser der FMH kann auf der Webseite des Departements «Ambulante Tarife und Verträge Schweiz» heruntergeladen werden. Dort erhalten Sie auch aktuelle Informationen. 


\section{Wann 00.0015 abrechnen?}

Grundsätzlich darf die neue Position nur von Fachärzten mit einem Weiterbildungstitel «Allgemeine Innere Medizin», «Kinder- und Jugendmedizin» oder «Praktischer Arzt» einmal pro Tag und Patient in Rechnung gestellt werden und, wie oben ausgeführt, nur dann, wenn ausschliesslich hausärztliche Leistungen erbracht werden.

Ob spezifische Leistungen als hausärztlich betrachtet werden können, muss, wie erwähnt, immer auch im Kontext ihrer Erbringung beurteilt werden. Hier besteht gemäss dem KVG auch eine Selbstverantwortung der abrechnungsberechtigten Hausärzte.

\section{Rechtliche Schritte gegen die Verordnung}

Verschiedene Organisationen, u.a. der Spitalverband $\mathrm{H}+$ und die fmCh, haben rechtliche Schritte gegen die Verordnung in die Wege geleitet. Über den aktuellen Stand der entsprechenden Rechtsverfahren werden Sie auf unserer Webseite informiert.

\section{Weitere Informationen}

Besuchen Sie die Internet-Seiten des Departements «Ambulante Tarif und Verträge Schweiz». Sie können dort auch einen aktuellen Tarifbrowser downloaden: www.fmh.ch/ambulante_tarife.html.

Wir informieren die Tarifdelegierten und Präsidenten der medizinischen Fachgesellschaften und

\title{
«Ein Tarifbrowser der FMH kann auf der Webseite des Departements 〈Ambulante Tarife und Verträge Schweiz〉 heruntergeladen werden.»
}

Als Leitlinie empfiehlt die FMH, sich an den aktuell im TARMED hinterlegten qualitativen Dignitäten zu orientieren (Leistungen, die mit einer hausärztlichen Dignität «Allgemeine Innere Medizin» bzw. «Kinder- und Jugendmedizin» oder als praktischer Arzt abgerechnet werden können). Ausserdem sollen Leistungen, die im Rahmen einer hausärztlichen Tätigkeit im Besitzstand oder mit einem Fähigkeitsausweis erbracht werden, ebenfalls mit der neuen Position kombiniert werden können. kantonalen Ärztegesellschaften per E-Mail und im «Infoletter Büro Tarife» über weitere Entwicklungen. (www.fmh.ch $\rightarrow$ Ambulante Tarife $\rightarrow$ Publikationen $\rightarrow$ Infoletter).

Bei Fragen und Problemen bei der Rechnungsstellung wenden Sie sich bitte telefonisch oder per E-Mail an die Abteilung «Ambulante Tarife und Verträge Schweiz».

\section{Wissen, was läuft.} Das News-Paket der FMH.

\author{
Schweizerische Ärztezeitung, Today's Press, \\ FMH-Flash. Für Mitglieder kostenlos.
}

